\title{
Psychotherapie bei Schizophrenie: Was geht?
}

\author{
Tania M. Lincoln ${ }^{a}$ Steffen Moritz ${ }^{b}$ \\ ${ }^{a}$ Arbeitsbereich Klinische Psychologie und Psychotherapie, Institut für Psychologie, Universität Hamburg, Hamburg, Deutschland; \\ ${ }^{\mathrm{b}}$ Klinik für Psychiatrie und Psychotherapie, Universitätsklinikum Hamburg-Eppendorf, Hamburg, Deutschland
}

Menschen mit psychotischen Störungen leiden unter einer Reihe von Problemen. Diese bieten sich für psychotherapeutische Interventionen geradezu an. Auch wünschen betroffene Patienten häufig eine psychotherapeutische Behandlung ihrer Probleme. Dazu gehören zum einen die Kernsymptome einer Schizophrenie, wie Verfolgungswahn, das Hören bedrohlicher Stimmen (Positivsymptomatik) oder der Verlust von Antrieb und Motivation (Negativsymptomatik). Zum anderen kann die Erfahrung einer akuten psychotischen Episode, die oft mit einem freiwilligen oder unfreiwilligen Krankenhausaufenthalt einhergeht, traumatisierend wirken. Häufig treten intrusive Erinnerungen an die psychotische Episode auf sowie eine anhaltende Sorge über mögliche Rückfälle. Ferner fehlen vielen Patienten mit Schizophrenie bestimmte basale Fertigkeiten, z.B. haben sie Schwierigkeiten, Probleme effektiv zu lösen, erfolgreich zu kommunizieren oder Gefühle effektiv zu regulieren. Diese Schwierigkeiten können die Wiedereingliederung in den beruflichen und sozialen Alltag erschweren. Auch für das soziale Umfeld ergeben sich häufig Probleme aus Unverständnis gegenüber wahnhafter Symptomatik oder aus Ungeduld gegenüber Motivationsmangel und Antriebsstörungen. Daher äußern auch viele Angehörige den Wunsch nach Begleitung und professioneller Unterstützung.

Erfreulicherweise gibt es inzwischen eine breite Palette psychotherapeutischer Ansätze, wenngleich wir weiter unten ein großes «Aber» anfügen. Weit verbreitet und im deutschsprachigen Raum schon seit 30 Jahren beforscht sind psychoedukative Familieninterventionen, die in nationalen und internationalen Leitlinien empfohlen werden [NCCMH, 2014]. Bei diesen Ansätzen steht die Aufklärung der Patienten und ihrer Angehörigen über die «Erkrankung» im Fokus. Das Ziel ist es, ein Krankheitsverständnis basierend auf Vulnerabilitäts-Stress-Modellen - zu erlangen und den selbstverantwortlichen Umgang mit der Erkrankung sowie ihre Bewältigung zu fördern. Eine federführende Rolle bei der

Korrespondenz kann ebenfalls gesendet werden an: Prof. Dr. Steffen Moritz, Klinik für Psychiatrie und Psychotherapie, Universitätsklinikum Hamburg-Eppendorf, Martinistraße 52, 20246 Hamburg, Deutschland, moritz@uke.uni-hamburg.de.
Entwicklung psychoedukativer Ansätze in Deutschland spielen Gabriele Pitschel-Walz und Josef Bäuml, die einen aktuellen Überblick über die Inhalte und den Forschungstand zur Evidenz sowie über die Wirkfaktoren psychoedukativer Verfahren geben [Pitschel-Walz und Bäuml, 2017]. Besonders interessant sind dabei neuere Entwicklungen zu kulturspezifischen Anpassungen psychoedukativer Programme für besondere Zielgruppen.

Ebenfalls auf eine etwa 30-jährige Tradition blicken verschiedene Skills-Training-Ansätze, die bei der Rezidivprophylaxe für schizophrene Patienten recht verbreitet sind. Basierend auf dem Vulnerabilitäts-Stress-Modell dienen solche Trainings vor allem dem Umgang mit Folgeproblemen der Störung, aber auch dem Aufbau von bereits prämorbid nicht vorhandenen Kompetenzen. Stellvertretend für den Bereich der Fertigkeitentrainings geben Daniel Müller und Volker Roder einen Überblick über den aktuellen Stand der Forschung zu kognitiven Trainings und Remediationsansätzen [Müller und Roder, 2017]. Diese Trainings zielen speziell auf die Modifikation kognitiver Defizite psychotischer Patienten durch eine Verbesserung kognitiver Funktionen. Dabei wird vor allem zwischen restitutiven (neue Fertigkeiten werden durch Üben gelernt) und kompensatorischen Ansätzen unterschieden. Die Autoren, die eine langjährige praktische und Forschungsexpertise in diesem Bereich aufweisen, beleuchten auch aktuellere Entwicklungen, die neben den klassischen Domänen Fertigkeiten im Bereich der sozialen Kognition mittrainieren.

Während Psychoedukation und Fertigkeitentrainings im deutschsprachigen Raum also bereits eine längere Behandlungstradition aufweisen, galten Interventionen, die unmittelbarer an den Symptomen ansetzen, bei Schizophrenie lange Zeit als kontraindiziert. In den klinisch-psychologischen Lehrbüchern wurde Schizophrenie für kognitiv-behaviorale Interventionen als Kontraindikation gelistet, und noch immer berichten uns junge Absolventen der Psychologie, sie hätten im Studium gelernt, dass man bei Schizophrenie psychotherapeutisch «nicht viel machen» könne. Auch in den Psychotherapie-Richtlinien wurden akut psychotische Patienten noch bis vor Kurzem von der Indikation ausgeschlossen, indem Psychotherapie hier auf die Behandlung der Residual-, Folge- oder Be-

\section{KARGER}

() 2017 S. Karger GmbH, Freiburg

Fax +497614520714
Prof. Dr. Tania M. Lincoln

Arbeitsbereich Klinische Psychologie und Psychotherapie, Institut für Psychologie Universität Hamburg

Von-Melle-Park 5, 20146 Hamburg, Deutschland

tania.lincoln@uni-hamburg.de 
gleitsymptomatik beschränkt wurde. Neben der sich hartnäckig haltenden Annahme, dass Schizophrenie fast ausschließlich durch biologische Faktoren verursacht wird, steckt hinter der Skepsis in Hinblick auf Psychotherapie bei diesem Störungsbild auch die Vorstellung, dass schizophrenietypische Symptome wie Wahn sich so stark vom normalen Erleben unterscheiden, dass sie für rationale Interventionen nicht zugänglich sind. Weit verbreitet ist die Befürchtung, Wahnsymptome könnten sich verschlimmern, wenn mit den Patienten über ihre Überzeugungen und Befürchtungen gesprochen wird. Diese Annahmen wurden jedoch in den letzten Jahrzehnten durch epidemiologische Studien relativiert. So wurde gezeigt, dass psychotisches Erleben auch in der Allgemeinbevölkerung nicht selten und eine klare Grenze zwischen gesund und krank schwer zu ziehen ist [van Os et al., 2009]. Stimmenhören kommt bei bis zu 15\% der Bevölkerung vor; nicht unmittelbar behandlungsbedürftige Wahnideen sind sogar noch stärker verbreitet. Wenn man aber davon ausgeht, dass es ein Kontinuum zwischen normalen und psychotischen Erlebnissen und Überzeugungen gibt und dass schizophrene Symptome somit lediglich extreme Manifestationen menschlichen Seins und Denkens sind, dann ist die Annahme folgerichtig, dass diese Erlebnisse und Symptome auch durch normale Prozesse der Meinungsbildung und des Lernens entstehen. Dies wiederum bildet eine wichtige Voraussetzung, um mit psychotherapeutischen Interventionen auch bei Schizophrenie anzusetzen. Ferner mehren sich Belege für soziale Risikofaktoren der Schizophrenie, wie Traumatisierung oder Migration [van Os et al., 2010], die - vermittelt über kognitive Schemata - mit psychotischen Symptomen assoziiert sind [Jaya et al., 2017]. Auch aus diesen Befunden ergeben sich vielversprechende Möglichkeiten für kognitive Ansätze.

Beflügelt durch diese Erkenntnisse hat die Kognitive Verhaltenstherapie (KVT) zur Behandlung von Wahn und Halluzinationen in den letzten Jahrzehnten großen Aufwind erhalten. Das wesentliche Ziel der KVT ist es, die Beeinträchtigung durch die Symptome zu lindern und das Funktionsniveau zu steigern. Dies geschieht vor allem durch den Aufbau von Verständnis für die Entstehung der Psychose, die Entlastung des Patienten durch eine entpathologisierende Haltung des Therapeuten und eine Korrektur dysfunktionaler Schemata, Annahmen und Coping-Strategien. Diese Ansätze sind vor allem von britischen Forschern [z.B. Fowler et al., 1995; Kingdon und Turkington, 2005] vorangetrieben worden und aufgrund ihrer Evidenzlage zur Reduktion von Positivsymptomatik und der Gesamtpsychopathologie inzwischen ein fester Bestandteil vieler Behandlungsleitlinien [z.B: NCCMH, 2014]. Neuere randomisiert-kontrollierte Studien weisen zudem darauf hin, dass KVT auch bei Patienten, die Medikamente verweigern, wirksam sein kann [Morrison et al., 2012]. Etwas ernüchternder sind die Wirksamkeitsbelege für KVT allerdings, wenn es um Patienten geht, bei denen eine Negativsymptomatik vorherrscht. Deshalb gehen Riehle et al. [2017] in diesem Heft der Frage nach, welche psychologischen Interventionen für diese Patientengruppe am ehesten Potenzial zeigen und weiterentwickelt werden sollten. Insgesamt deutet die Studienlage darauf hin, dass Negativsymptomatik durchaus «behandelbar» ist, allerdings bedarf es verstärkter Forschungsbemühungen in diesem Bereich in Richtung eines dif- ferenzierteren Verständnisses der Symptomatik und ihrer auslösenden und aufrechterhaltenden Faktoren und vermutlich eines stärker individualisierten Vorgehens.

Aufgrund der neuen Evidenzlage passte der Gemeinsame Bundesausschuss die Psychotherapie-Richtlinie im Oktober 2014 an und erklärte Psychotherapie bei Schizophrenie sowie schizotypen und wahnhaften Störungen für uneingeschränkt indiziert. Hier setzt das oben angekündigte «Aber» ein. Trotz dieser erfreulichen Entwicklungen schildern Patienten immer wieder, dass sie von ambulanten Psychotherapeuten zurückgewiesen werden. Auch verschiedene Studien legen nahe, dass die Empfehlungen der Behandlungsleitlinien in der klinischen Praxis noch nicht hinreichend ankommen. Das gilt vor allem für ambulante Psychotherapie [Bechdolf und Klingberg, 2014; Schlier und Lincoln, 2016], aber auch für stationäre Angebote (siehe z.B. Pitschel-Walz und Bäuml [2017] in diesem Heft). Um diesem Missstand zu begegnen, bedarf es aus unserer Sicht zunächst eines besseren Verständnisses davon, an welcher Stelle Behandlungshürden entstehen. In diesem Sinne gehen Schlier et al. [2017] im vorliegenden Heft mit einer «Verdeckter-Ermittler-Studie» der Frage nach, ob Patienten mit Schizophrenie auf der Suche nach einem Therapieplatz diskriminiert werden.

Während einerseits Behandlungsbarrieren für die ambulante Versorgung abgebaut werden müssen, stellt sich andererseits auch die Frage nach niedrigschwelligen Behandlungsformen. In der Tat verlangt der Ansatz der Umstrukturierung von Wahn dem Therapeuten ein hohes Maß an Fingerspitzengefühl und Erfahrung ab, da einerseits der Patient mit seiner Sicht der Dinge verstanden und gewürdigt, andererseits aber der Wahn des Patienten kritisch hinterfragt werden soll [Lincoln und Heibach, im Druck]. Aus praktisch-therapeutischer Perspektive wäre daher eine Alternative zur kognitiven Umstrukturierung von Wahn wünschenswert, die einfacher zu erlernen und zu handhaben ist und weniger Gefahr birgt, die Patient/Therapeut-Beziehung zu belasten. Ansatzpunkte für spezifische Interventionen, die das Potenzial zur Reduktion von Wahn haben und für Therapeuten und Patienten akzeptabler sein könnten, lassen sich den Befunden aus der klinischen Grundlagenforschung zu emotionalen [Lincoln et al., 2015] und kognitiven Prozessen [Savulich et al., 2012] der Wahnentstehung entnehmen. Um einen therapeutischen Ansatz, der auf sogenannte kognitive Verzerrungen bei der Wahnentstehung fokussiert, geht es in dem Beitrag von Dietrichkeit et al. [2017]. Dieser Ansatz versucht, kognitive Verzerrungen wie voreiliges Schlussfolgern, Inflexibilität und Überkonfidenz zu «begradigen», indem Patienten spielerisch die Dysfunktionalität dieser Denkstile vor Augen geführt wird. Zentral ist bei diesem Ansatz das Element der Normalisierung, indem demonstriert wird, dass die meisten Menschen Denkverzerrungen aufweisen, die bei abnormer Steigerung zu psychischen Problemen führen können. Dies entlastet viele Patienten, da die Prozesse, die eine Psychose verursachen können, als allgemeinmenschlich vermittelt werden, und fördert so ein psychologisches Verständnis der Entstehung und Aufrechterhaltung von Psychosen. Eine neuere Meta-Analyse bestätigte die Wirksamkeit dieses Ansatzes [Eichner und Berna, 2016], wenngleich die Intervention für hochakute Patienten nur bedingt geeignet scheint. 
Eine ganz andere Antwort auf die psychotherapeutische Unterversorgung von Schizophreniepatienten könnte in einem Ausbau verschiedener Online-Interventionen liegen. Westermann et al. [2017] beleuchten, wie verschiedene Formen von Internet- und mobilbasierten Interventionen das bestehende Behandlungsangebot für Patienten mit psychotischen Störungen ergänzen können. Online-Interventionen können insbesondere bei der Psychoedukation helfen, da in den standardisierten Basisprogrammen zusätzliche Bereiche, wie z.B. Schlafhygiene, oft zu kurz kommen [siehe Pitschel-Walz und Bäuml, 2017].

Der weite Weg, den die Psychotherapie der Psychosen in kurzer Zeit zurückgelegt hat, ist beeindruckend. Galten noch bis in die 2000er Jahre hinein medikamentöse Ansätze als Grundsäule der Behandlung, sind psychotherapeutische Ansätze inzwischen aufgrund ihrer Evidenz in einigen Leitlinien als gleichwertige Säule verankert. Dennoch sind wir von der Zielvorstellung, möglichst allen Patienten mit psychotischen Störungen eine evidenzbasierte Psychotherapie anbieten zu können, noch recht weit entfernt. Eine Behandlungsbarriere scheint in einem Mangel an qualifizierten Fortbildungen und fallbezogener Supervision zu liegen. Ferner wäre eine engere Verzahnung von stationärer und ambulanter Therapie wünschenswert, die auch die psychotherapeutische Begleitung und Aufarbeitung von Rückfällen erleichtern würde, vor allem, wenn ein gestufter Wiedereinstieg in eine ambulante Behandlung abrechenbar wäre. Gingen solche strukturellen Veränderungen zudem Hand in Hand mit einem veränderten Bild von Psychosen, frei von stigmatisierenden Zuschreibungen oder Mythen, wäre eine deutlich verbesserte Versorgungssituation für Patienten mit Psychosen möglich. Wir hoffen, diesem Ziel in den nächsten Jahren ein gutes Stück näherzukommen und mit diesem Themenheft zeigen zu können, dass die Psychotherapie von Psychosen sich lohnt.

\section{Literatur}

Bechdolf A, Klingberg S: Psychotherapie bei schizophrenen Störungen: Kein Evidenz-, sondern ein Implementierungsproblem. Psychiatrische Praxis 2014;41:8-10.

Dietrichkeit M, Krieger E, Kolbeck K, Moritz S: Behandlungen kognitiver Verzerrungen bei Psychose: Ein Überblick der aktuellen Befunde. Verhaltenstherapie 2017;27:doi: 10.1159/000464340.

Eichner C, Berna F: Acceptance and Efficacy of Metacognitive Training (MCT) on Positive Symptoms and Delusions in Patients With Schizophrenia: A Meta-analysis Taking Into Account Important Moderators. Schizophr Bull 2016;42:952-962.

Fowler D, Garety P, Kuipers E: Cognitive Behaviour Therapy for Psychosis: Theory and Practice. Chichester, Wiley, 1995

Jaya ES, Ascone L, Lincoln TM: Social Adversity and Psychosis: The Mediating Role of Cognitive Vulnerability. Schizophr Bull 2017;43:557-565.

Kingdon DG, Turkington D: Cognitive Therapy of Schizophrenia. New York, Guilford Press, 2005.

Lincoln T, Heibach E: Psychosen, vol 67. Göttingen, Hogrefe, im Druck.

Lincoln TM, Hartmann M, Köther U, Moritz S: Do people with psychosis have specific difficulties regulating emotions? Clin Psychol Psychother 2015;22:637-646.
Morrison AP, Hutton P, Wardle M, et al: Cognitive therapy for people with a schizophrenia spectrum diagnosis not taking antipsychotic medication: an exploratory trial. Psychol Med 2012;42:1049-1056.

Müller DR, Roder V: Vom kognitiven Training zur Kognitiven Remediationstherapie: Nutzen und Grenzen. Verhaltenstherapie 2017;27:doi: 10.1159/000479853.

NCCMH (National Collaborating Centre for Mental Health): Psychosis and schizophrenia in adults. The NICE guideline on treatment and management. NICE Clinical Guideline 178. London, NICE, 2014.

Pitschel-Walz G, Bäuml J: Psychoedukation für Patienten und Angehörige: Eine psychotherapeutische Basisbehandlung bei schizophrenen Psychosen. Verhaltenstherapie 2017;27:doi: 10.1159/000478912.

Riehle M, Pillny M, Lincoln TM: Ist Negativsymptomatik bei Schizophrenie überhaupt behandelbar? Ein systematisches Literaturreview zur Wirksamkeit psychotherapeutischer Interventionen für Negativsymptomatik. Verhaltenstherapie 2017;27:doi: 10.1159/000478534.

Savulich G, Shergill S, Yiend J: Biased Cognition in Psychosis. J Exp Psychopathol 2012;3:514-536.
Schlier B, Lincoln TM: Blinde Flecken? Der Einfluss von Stigma auf die psychotherapeutische Versorgung von Menschen mit Schizophrenie. Verhaltenstherapie 2016;26:279-290.

Schlier B, Wiese S, Frantz I, Lincoln TM: Chancengleichheit in der ambulanten Therapie: Ein Experiment zur Bereitschaft von niedergelassenen Psychotherapeuten, Patienten mit Schizophrenie zu behandeln. Verhaltenstherapie 2017;27:doi: 10.1159/000478533.

van Os J, Kenis G, Rutten BP: The environment and schizophrenia. Nature 2010;468:203-212.

van Os J, Linscott RJ, Myin-Germeys I, et al: A systematic review and meta-analysis of the psychosis continuum: evidence for a psychosis proneness-persistenceimpairment model of psychotic disorder. Psychol Med 2009;39:179-195.

Westermann S, Moritz S, Berger T: Internet- und mobilbasierte Interventionen bei Schizophrenie. Verhaltenstherapie 2017;27:doi: 10.1159/000479335. 